Atmospheric Chemistry and Physics

Supplemental Information for

\author{
A New Model of Meteoric Calcium in the Mesosphere and Lower Thermosphere \\ John M. C. Plane ${ }^{1}$, Wuhu Feng ${ }^{1,2}$, Juan Carlos Gómez Martín ${ }^{1,3}$, Michael Gerding ${ }^{4}$, and Shikha \\ Raizada $^{5}$ \\ ${ }^{1}$ School of Chemistry, University of Leeds, Leeds LS2 9JT, U.K. \\ ${ }^{2}$ National Centre for Atmospheric Science and School of Earth and Environment, University of Leeds, Leeds LS2 9JT, \\ UK. \\ ${ }^{3}$ Instituto de Astrofísica de Andalucía (IAA-CSIC), 18008 Granada, Spain. \\ ${ }^{4}$ Leibniz Institute of Atmospheric Physics, Rostock University, Schlossstraße 6, 18225 Kühlungsborn, Germany. \\ ${ }^{5}$ Space and Atmospheric Science Department, Arecibo Observatory/SRI International, Arecibo 00612, Puerto Rico.
}

\title{
Contents of this file
}

Text $\mathrm{S} 1$ to $\mathrm{S} 3$

Figures $\mathrm{S} 1$ to $\mathrm{S} 4$

Tables S1 to S5

\section{Introduction}

Text $\mathrm{S} 1$ describes the method used to calculate the rate coefficient for the reaction between $\mathrm{CaCO}_{3}$ and $\mathrm{O}$, using the molecular parameters listed in Table $\mathrm{S} 1$. The potential energy surface for this reaction is shown in Figure S1. Text S2 describes the method used to calculate the rate coefficient for the reaction between $\mathrm{CaCO}_{3}$ and $\mathrm{O}_{2}$, using the molecular parameters listed in Table S2. Text S3 describes the method used to calculate the rate coefficient for the reaction between $\mathrm{O}_{2} \mathrm{CaCO}_{3}$ and $\mathrm{O}$, using the molecular parameters listed in Table S3. The potential energy surface for this reaction is shown in Figure $2 b$ in the main paper.

Figure $\mathrm{S} 2$ shows the vertically integrated $\mathrm{Na}$ and $\mathrm{Ca}$ meteoric input fluxes used in WACCM-Na and WACCM-Ca, plotted as a function of latitude and month. Figure S3 shows the monthly mean RMS (root-mean-square) width and centroid height of the Ca layer from WACCM-Ca, plotted as a function of latitude and month.

Figure $\mathrm{S} 4$ compares $\mathrm{Na}^{+}$and $\mathrm{Ca}^{+}$density profiles measured by rocket-borne mass spectrometry, compared with WACCM, at a selection of latitudes and seasons. Figure S5 
compares the measured and modeled Na column abundance as a function of latitude and month.

Table S4 lists the monthly mean Ca atom column abundance, as a function of latitude and month. Table S5 lists the monthly mean $\mathrm{Ca}^{+}$ion column abundance, as a function of latitude and month.

\section{Text S1. The reaction between $\mathrm{CaCO}_{3}$ and $\mathrm{O}$}

The rate coefficient for reaction $\mathrm{R} 16$ in the paper

$$
\mathrm{CaCO}_{3}+\mathrm{O} \rightarrow \mathrm{CaO}_{2}+\mathrm{CO}_{2}
$$

was calculated using transition state theory (TST). As shown in Figure S1, there is a deep well before the barrier where the $\mathrm{O}$ adds to the $\mathrm{Ca}$. There is then a substantial barrier (33 $\mathrm{kJ} \mathrm{mol}^{-1}$ ) to formation of the products. The data required for the calculation is listed in Table S1, and the rate coefficient is $k_{16}=4.0 \times 10^{-12} \exp (-4689 / T) \mathrm{cm}^{3}$ molecule $^{-1} \mathrm{~s}^{-1}$.

Note that although $\mathrm{OCaCO}_{3}$ formation should be a reasonably fast recombination reaction, it will not compete with addition of $\mathrm{O}_{2}$ because the concentration of $\mathrm{O}_{2}$ is at least $10^{3}$ times larger below $85 \mathrm{~km}$.

Table S1. Molecular properties of the stationary points on the potential energy surface for $\mathrm{CaCO}_{3}+\mathrm{O}$ (Figure S1), calculated at the B3LYP/6-311+g(2d,p) level of theory with the Gaussian suite of programs [Frisch et al., 2009].

\begin{tabular}{|c|c|c|c|}
\hline Molecule & $\begin{array}{l}\text { Geometry (Cartesian co- } \\
\text { ordinates in } \AA \text { ) }\end{array}$ & $\begin{array}{l}\text { Rotational } \\
\text { constants }(\mathrm{GHz})\end{array}$ & $\begin{array}{l}\text { Vibrational frequencies } \\
\left(\mathrm{cm}^{-1}\right)\end{array}$ \\
\hline $\mathrm{CaCO}_{3}$ & $\begin{array}{l}\mathrm{Ca}, 0.0,0.0,0.0 \\
\mathrm{O}, 0.0,0.0,2.052 \\
\mathrm{O}, 1.876,0.0,0.832 \\
\mathrm{O}, 2.016,0.0,3.100 \\
\mathrm{C}, 1.357,0.0,2.087\end{array}$ & $\begin{array}{l}12.621 .2 .806, \\
2.296\end{array}$ & $\begin{array}{l}120,363,453 \\
656,762,828 \\
951,1084,1752\end{array}$ \\
\hline $\mathrm{OCaCO}_{3}$ & $\begin{array}{l}\mathrm{Ca}, 0.0,1.094,0.0 \\
\mathrm{O},-1.105,-0.966,0.0 \\
\mathrm{O}, 1.128,-0.954,-0.005 \\
\mathrm{O}, 0.022,-2.851,0.007 \\
\mathrm{C}, 0.015,-1.560,-0.002 \\
\mathrm{O},-0.010,3.084,-0.698\end{array}$ & $\begin{array}{l}11.626,1.278, \\
1.171\end{array}$ & $\begin{array}{l}26,59,85,233,277 \\
381,521,668,821 \\
1041,1323,1462\end{array}$ \\
\hline $\begin{array}{l}\text { TS to } \\
\text { products } \\
\mathrm{CaO}_{2}+\mathrm{CO}_{2}\end{array}$ & $\begin{array}{l}\mathrm{Ca}, 0.0,0.0,0.0 \\
\mathrm{O}, 0.0,0.0,2.229 \\
\mathrm{O}, 2.035,0.0,0.431 \\
\mathrm{O}, 1.939,0.003,3.467 \\
\mathrm{C}, 1.219,0.002,2.530 \\
\mathrm{O}, 1.814,-0.002,-1.161\end{array}$ & $\begin{array}{l}5.342,1.856, \\
1.378\end{array}$ & $\begin{array}{l}722 i, 65,118,184,211, \\
332,407,416,577, \\
701,1182,1937\end{array}$ \\
\hline
\end{tabular}




\section{Text S2. The reaction between $\mathrm{CaCO}_{3}$ and $\mathrm{O}_{2}$}

The rate coefficient for the recombination reaction

$$
\mathrm{CaCO}_{3}+\mathrm{O}_{2}(+\mathrm{M}) \rightarrow \mathrm{O}_{2} \mathrm{CaCO}_{3} \quad \Delta H^{\mathrm{o}}=-89 \mathrm{~kJ} \mathrm{~mol}^{-1}
$$

was calculated using Rice-Ramsperger-Kassel-Marcus Theory - see Gómez-Martín and Plane [2017] for further details. Using the molecular parameters in Table S2, the rate coefficient is estimated to be:

$$
\begin{aligned}
& k_{0}(200 \mathrm{~K})=4.0 \times 10^{-26}(T / 200)^{-3.85} \mathrm{~cm}^{6} \text { molecule } \mathrm{s}^{-1} \\
& k_{\infty}(200 \mathrm{~K})=5.9 \times 10^{-10} \exp (-46 / T) \mathrm{cm}^{3} \text { molecule } \mathrm{s}^{-1} \mathrm{~s}^{-1} \\
& F_{\mathrm{c}}=0.33
\end{aligned}
$$

where these three parameters required to calculate the recombination rate in the fall-off region are described in Gómez-Martín and Plane [2017]. The recombination rate constant at $200 \mathrm{~K}$ and $[\mathrm{M}]=1 \times 10^{14} \mathrm{~cm}^{-3}$ (i.e. around $84 \mathrm{~km}$ ), is $5.9 \times 10^{-12} \mathrm{~cm}^{3}$ molecule $^{-1} \mathrm{~s}^{-1}$.

Table S2. Molecular properties of the stationary points on the potential energy surface for $\mathrm{CaCO}_{3}+\mathrm{O}_{2}$ (see Figure $2 \mathrm{a}$ for geometries of $\mathrm{CaCO}_{3}$ and $\mathrm{O}_{2} \mathrm{CaCO}_{3}$ ), calculated at the B3LYP/6-311+g(2d,p) level of theory with the Gaussian suite of programs [Frisch et al., 2009].

\begin{tabular}{|l|l|l|l|}
\hline Molecule & $\begin{array}{l}\text { Geometry }(\text { Cartesian co- } \\
\text { ordinates in } \AA)\end{array}$ & $\begin{array}{l}\text { Rotational } \\
\text { constants }(\mathrm{GHz})\end{array}$ & $\begin{array}{l}\text { Vibrational frequencies } \\
\left(\mathrm{cm}^{-1}\right)\end{array}$ \\
\hline $\mathrm{CaCO}_{3}$ & $\mathrm{Ca}, 0.0,0.0,0.0$ & 12.621 .2 .806$, & $120,363,453$, \\
& $\mathrm{O}, 0.0,0.0,2.052$ & 2.296 & $656,762,828$, \\
& $\mathrm{O}, 1.876,0.0,0.832$ & & $951,1084,1752$ \\
& $\mathrm{O}, 2.016,0.0,3.100$ & & \\
& $\mathrm{C}, 1.357,0.0,2.087$ & & $41,45,48,90,236$, \\
& $\mathrm{Ca},-0.737,-0.0,0.020$ & $9.321,0.905$, & $250,374,408,470$, \\
$\mathrm{O}_{2} \mathrm{CaCO}_{3}$ & $\mathrm{O}, 1.251,1.222,0.088$ & 0.865 & $674,820,1038,1183$, \\
& $\mathrm{O},-2.846,-0.146,0.679$ & & 1341,1472 \\
& $\mathrm{O},-2.843,-0.084,-0.658$ & & \\
& $\mathrm{O}, 3.199,0.210,0.048$ & & \\
& $\mathrm{O}, 1.370,-1.005,-0.019$ & & \\
& $\mathrm{C}, 1.908,0.1405,0.0390$ & & \\
& & & \\
\end{tabular}




\section{Text S3. The reaction between $\mathrm{O}_{2} \mathrm{CaCO}_{3}$ and $\mathrm{O}$}

The reactants $\mathrm{O}_{2} \mathrm{CaCO}_{3}$ and $\mathrm{O}$ are both triplets, so this reaction can take place on singlet, triplet and quintet surfaces. However, electronic structure calculations show that the quintet surface is unreactive. The potential energy surface (Figure 2b) shows the pathways on the singlet and triplet surfaces. The molecular parameters of the stationary points are listed in Table S3.

Table S3. Molecular properties of the stationary points on the potential energy surface for $\mathrm{O}_{2} \mathrm{CaCO}_{3}+\mathrm{O}_{2}$ (see Figure 2b), calculated at the B3LYP/6-311+g(2d,p) level of theory with the Gaussian suite of programs [Frisch et al., 2009].

\begin{tabular}{|c|c|c|c|}
\hline Molecule & $\begin{array}{l}\text { Geometry (Cartesian co-ordinates } \\
\text { in } \AA \text { ) }\end{array}$ & $\begin{array}{l}\text { Rotational constants } \\
(\mathrm{GHz})\end{array}$ & $\begin{array}{l}\text { Vibrational } \\
\text { frequencies } \\
\left(\mathrm{cm}^{-1}\right)\end{array}$ \\
\hline $\mathrm{O}_{2} \mathrm{CaCO}_{3}$ & $\begin{array}{l}\mathrm{Ca},-0.737,-0.0,0.020 \\
\mathrm{O}, 1.251,1.222,0.088 \\
\mathrm{O},-2.846,-0.146,0.679 \\
\mathrm{O},-2.843,-0.084,-0.658 \\
\mathrm{O}, 3.199,0.210,0.048 \\
\mathrm{O}, 1.370,-1.005,-0.019 \\
\mathrm{C}, 1.908,0.1405,0.0390\end{array}$ & $9.321,0.905,0.865$ & $\begin{array}{l}41,45,48,90, \\
236,250,374, \\
408,470,674, \\
820,1038, \\
1183,1341, \\
1472\end{array}$ \\
\hline $\begin{array}{l}\mathrm{O}_{3} \mathrm{CaCO}_{3} \\
\text { triplet }\end{array}$ & $\begin{array}{l}\mathrm{Ca},-0.372,0.143,-0.071 \\
\mathrm{O}, 1.700,-0.905,0.039 \\
\mathrm{O}, 1.632,1.320,-0.130 \\
\mathrm{O}, 3.558,0.267,-0.021 \\
\mathrm{C}, 2.266,0.226,-0.038 \\
\mathrm{O},-3.111,0.059,-0.105 \\
\mathrm{O},-2.379,0.164,1.029 \\
\mathrm{O},-2.346,0.001,-1.221\end{array}$ & $6.274,0.690,0.689$ & $\begin{array}{l}37,43,45,93, \\
153,218,238, \\
304,375,386, \\
661,678,819, \\
877,1039, \\
1083,1347, \\
1473\end{array}$ \\
\hline $\begin{array}{l}\mathrm{O}_{3} \mathrm{CaCO}_{3} \\
\text { singlet }\end{array}$ & $\begin{array}{l}\mathrm{Ca},-0.281,-0.812,0.301 \\
\mathrm{O}, 1.849,-1.023,-0.063 \\
\mathrm{O}, 0.998,1.021,0.019 \\
\mathrm{O}, 3.191,0.752,-0.196 \\
\mathrm{C}, 2.057,0.271,-0.090 \\
\mathrm{O},-2.702,0.580,-0.251 \\
\mathrm{O},-2.092,0.644,0.907 \\
\mathrm{O},-2.072,-0.157,-1.142\end{array}$ & $4.158,0.868,0.809$ & $\begin{array}{l}22,36,60,98, \\
199,238,258, \\
307,339,558, \\
660,712,831, \\
1011,1037, \\
1106,1255, \\
1519\end{array}$ \\
\hline TS1 & $\begin{array}{l}\mathrm{Ca},-0.722,0.445,-0.147 \\
\mathrm{O}, 1.319,-0.189,-1.104 \\
\mathrm{O}, 1.271,0.306,1.074 \\
\mathrm{O}, 3.140,-0.330,0.114 \\
\mathrm{C}, 1.878,-0.063,0.026 \\
\mathrm{O},-3.334,-1.705,0.284 \\
\mathrm{O},-2.127,-1.781,0.327\end{array}$ & $2.611,0.776,0.661$ & $\begin{array}{l}94 i, 25,26,36, \\
63,76,86, \\
156,232,279, \\
359,516,672, \\
820,1038, \\
1338,1469, \\
1572\end{array}$ \\
\hline
\end{tabular}




\begin{tabular}{|c|c|c|c|}
\hline & $\mathrm{O},-2.383,1.711,-0.471$ & & \\
\hline TS2 & $\begin{array}{l}\text { Ca, }-0.148,-0.138,-0.005 \\
\mathrm{O}, 1.726,-0.221,-1.075 \\
\mathrm{O}, 1.649,0.457,1.034 \\
\mathrm{O}, 3.607,0.402,-0.042 \\
\mathrm{C}, 2.389,0.221,-0.0281 \\
\mathrm{O},-3.683,-0.439,-0.047 \\
\mathrm{O},-4.136,-1.562,0.297 \\
\mathrm{O},-2.362,-0.3262,-0.0312\end{array}$ & $9.983,0.512,0.498$ & $\begin{array}{l}27 i, 20,35,77, \\
114,149,213, \\
331,371,594, \\
676,702,834, \\
980,1029, \\
1222,1222, \\
1542\end{array}$ \\
\hline TS3 & $\begin{array}{l}\mathrm{Ca},-0.592,-1.342,-0.049 \\
\mathrm{O}, 1.610,-1.030,-0.680 \\
\mathrm{O}, 0.368,0.501,0.781 \\
\mathrm{O}, 2.779,0.729,0.225 \\
\mathrm{C}, 1.925,-0.012,-0.072 \\
\mathrm{O},-1.853,1.044,0.142 \\
\mathrm{O},-2.422,-0.266,0.0581 \\
\mathrm{O},-0.511,0.987,-0.336\end{array}$ & $3.329,1.276,0.983$ & $\begin{array}{l}161 i, 66,100, \\
146,230,267, \\
315,396,433, \\
494,534,625, \\
678,716,819, \\
917,1251, \\
2089\end{array}$ \\
\hline $\begin{array}{l}\mathrm{O}_{2} \mathrm{CaO}_{2} \\
\text { singlet }\end{array}$ & $\begin{array}{l}\mathrm{Ca}, 0.094,1.075,-0.005 \\
\mathrm{O},-1.710,-0.186,-0.260 \\
\mathrm{O}, 1.734,2.427,-0.635 \\
\mathrm{O}, 1.1713,2.954,0.457 \\
\mathrm{O},-0.850,-0.863,0.508\end{array}$ & $17.670,1.686,1.686$ & $\begin{array}{l}42,42,151, \\
347,386,414, \\
414,1084, \\
1174\end{array}$ \\
\hline $\begin{array}{l}\mathrm{OCaCO}_{3} \\
\text { triplet }\end{array}$ & $\begin{array}{l}\mathrm{Ca}, 0.001,1.094,0.001 \\
\mathrm{O},-1.105,-0.966,-0.004 \\
\mathrm{O}, 1.128,-0.954,-0.005 \\
\mathrm{O}, 0.022,-2.851,0.007 \\
\mathrm{C}, 0.015,-1.560,-0.002 \\
\mathrm{O},-0.010,3.084,-0.698\end{array}$ & $11.626,1.278,1.171$ & $\begin{array}{l}26,59,85, \\
233,277,381, \\
521,668,821, \\
1041,1323, \\
1462\end{array}$ \\
\hline
\end{tabular}




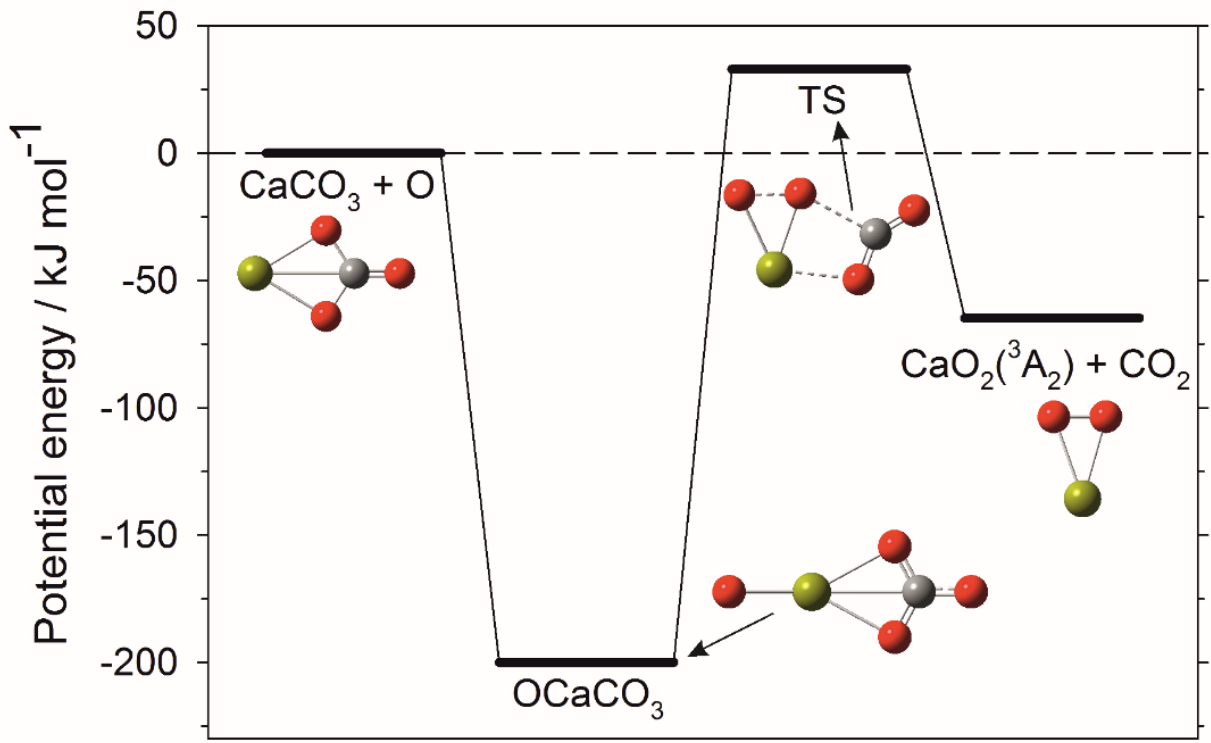

Reaction coordinate

Figure S1. Potential energy surface for the reaction between $\mathrm{CaCO}_{3}$ and $\mathrm{O}$, calculated at the B3LYP/6-311+g(2d,p) level of theory [Frisch et al., 2009]. Colour scheme: Ca (yellow); C (grey); O (red). 

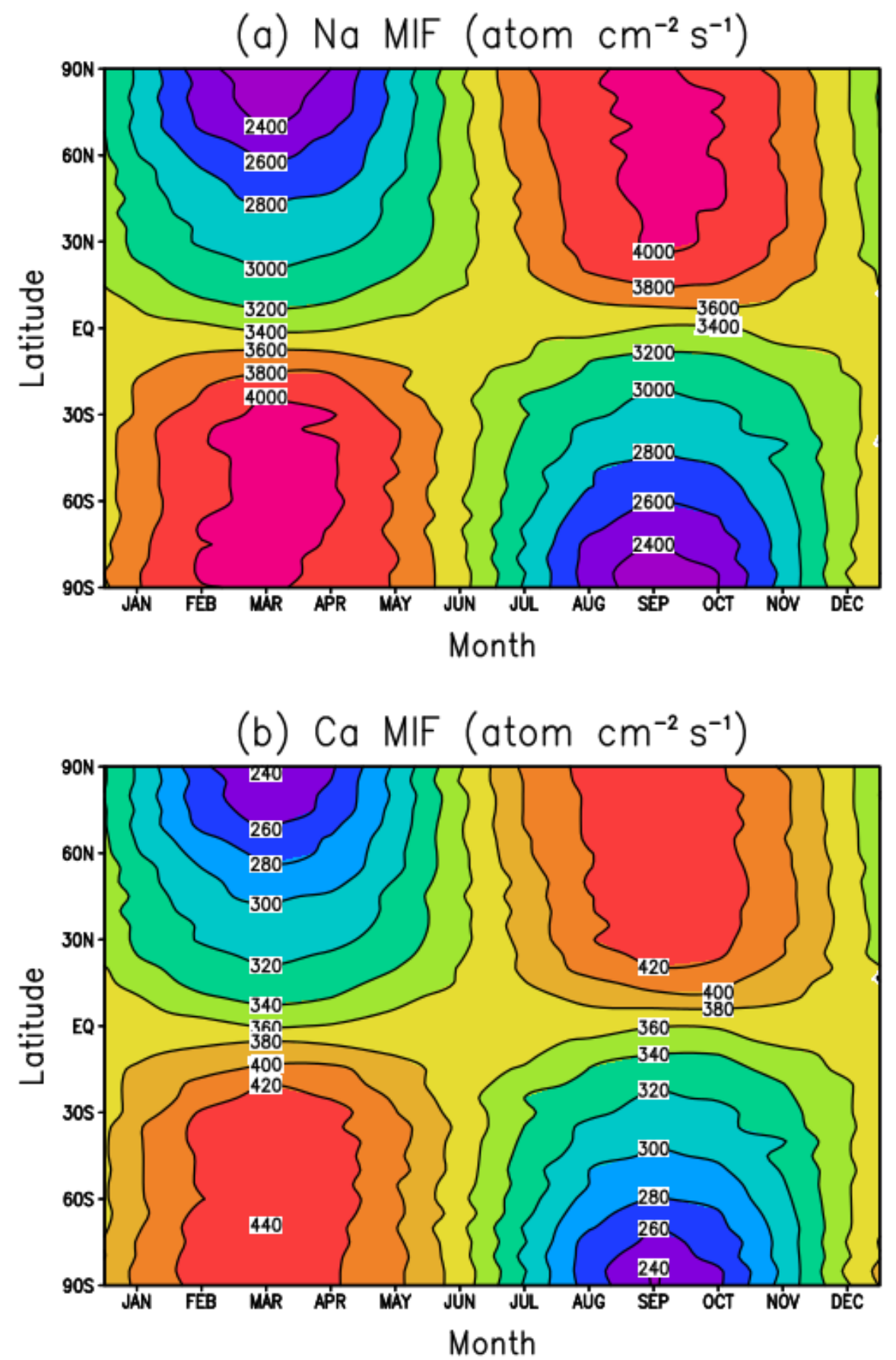

Figure S2. Vertically integrated $\mathrm{Na}$ and Ca meteoric input fluxes (units: atom $\mathrm{cm}^{-2} \mathrm{~s}^{-1}$ ) used in WACCM-Na and WACCM-Ca, plotted as a function of latitude and month. 

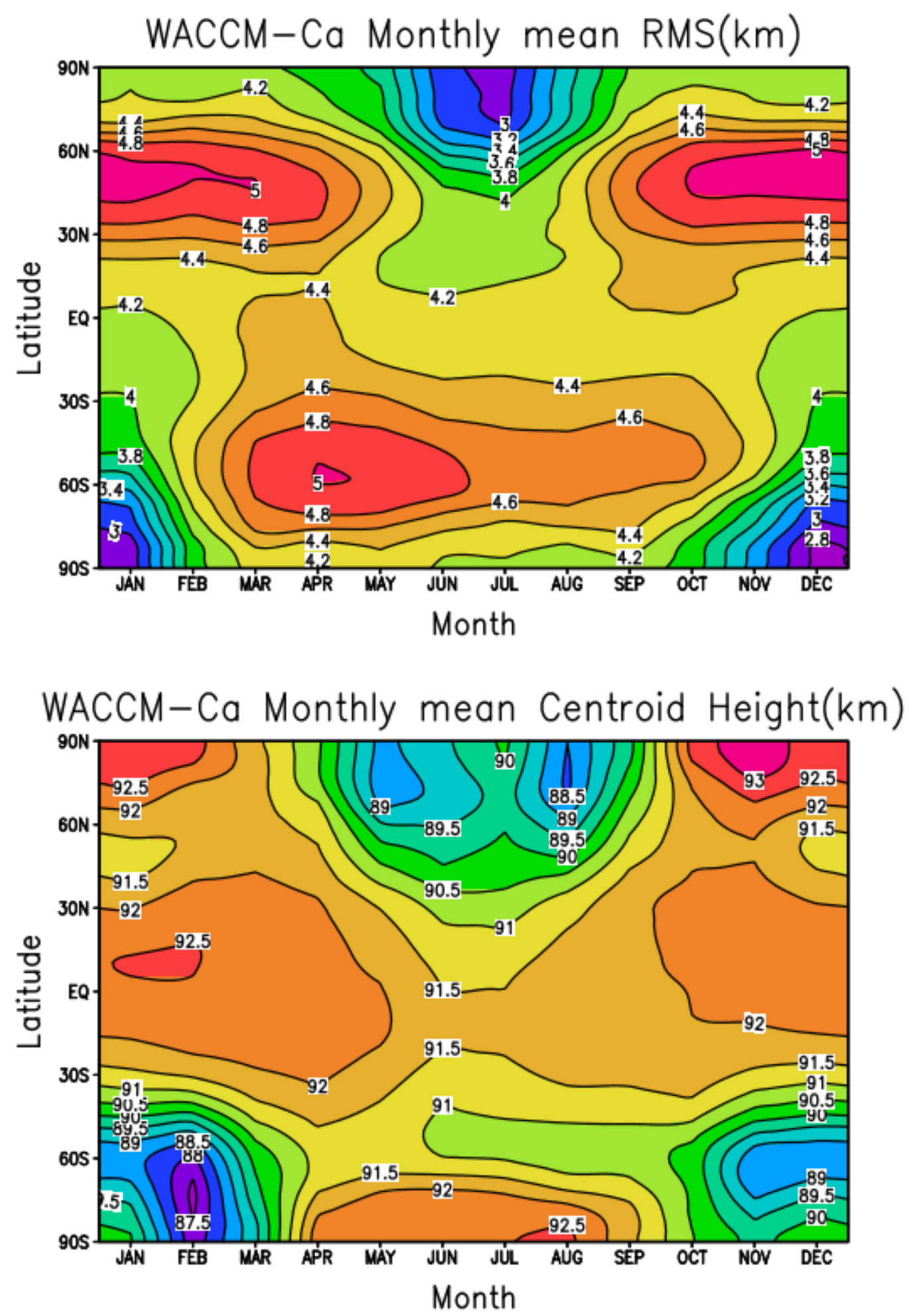

Figure S3. Monthly mean RMS width and centroid height (units: $\mathrm{km}$ ) of the Ca layer from WACCM-Ca, plotted as a function of latitude and month. 


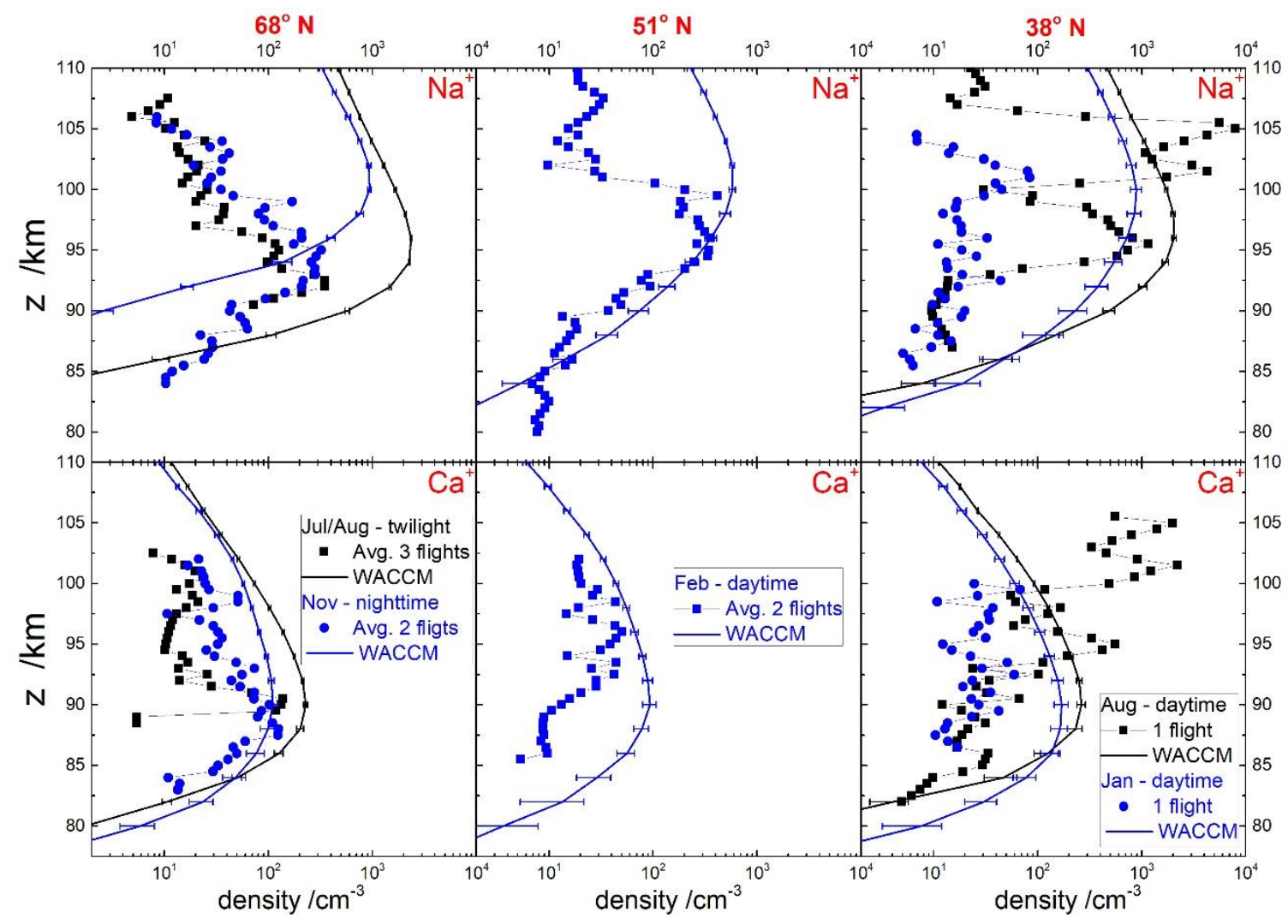

Figure S4. Latitudinal and seasonal variability of observed (symbols) and modelled (solid lines) $\mathrm{Na}^{+}$and $\mathrm{Ca}^{+}$density profiles (top and bottom panel rows respectively). The datasets are grouped in panel columns by the latitude of where the rocket-borne mass spectrometric measurements took place (from left to right $68^{\circ} \mathrm{N}, 51^{\circ} \mathrm{N}$ and $38^{\circ} \mathrm{N}$ ). The ion density profiles are averaged by month or season (summer: black; fall/winter: blue). 

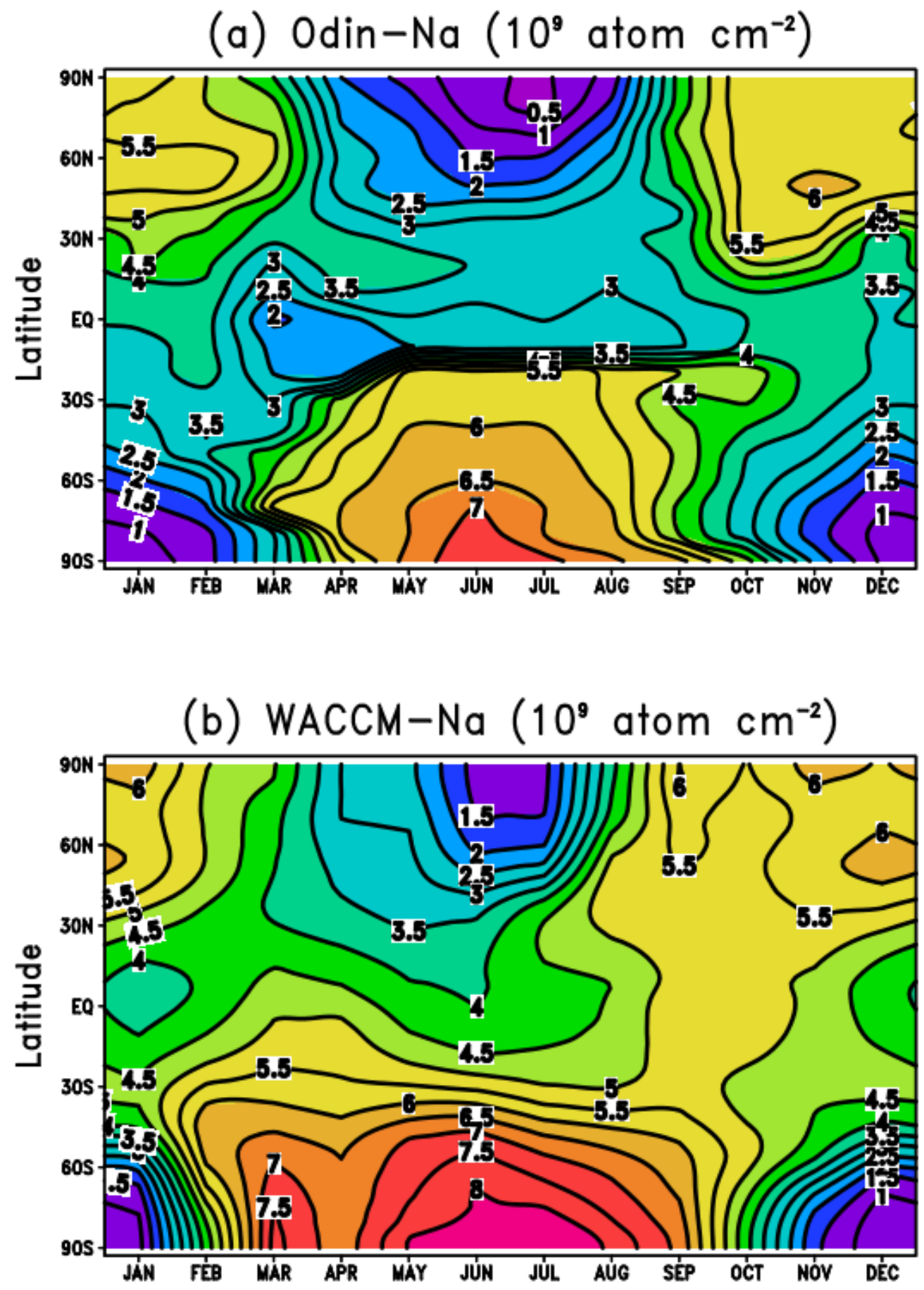

Figure S5. Diurnally averaged column abundance of the mesospheric Na layer, plotted as a function of latitude and month: (a) from a Na climatology [Dawkins et al., 2015]; (b) calculated by the WACCM-Na model with revised rate coefficients and the new $\mathrm{Na}$ meteoric input function. 
Table S4. Monthly mean Ca column abundance $(70-110 \mathrm{~km})$ predicted by WACCM-Ca (units: $10^{7}$ atom $\mathrm{cm}^{-2}$ )

\begin{tabular}{|c|c|c|c|c|c|c|c|c|c|c|c|c|}
\hline Latitude & Jan & Feb & Mar & Apr & May & Jun & Jul & Aug & Sep & Oct & Nov & Dec \\
\hline-90.00 & 0.64 & 1.51 & 2.43 & 3.35 & 4.95 & 5.15 & 4.91 & 3.73 & 1.83 & 1.15 & 0.72 & 0.52 \\
\hline-88.11 & 0.64 & 1.52 & 2.43 & 3.32 & 4.92 & 5.14 & 4.89 & 3.69 & 1.83 & 1.15 & 0.72 & 0.52 \\
\hline-86.21 & 0.64 & 1.53 & 2.46 & 3.26 & 4.86 & 5.11 & 4.87 & 3.61 & 1.85 & 1.15 & 0.72 & 0.52 \\
\hline-84.32 & 0.65 & 1.56 & 2.5 & 3.18 & 4.78 & 5.07 & 4.82 & 3.5 & 1.89 & 1.15 & 0.74 & 0.53 \\
\hline-82.42 & 0.65 & 1.58 & 2.54 & 3.09 & 4.68 & 5.02 & 4.74 & 3.37 & 1.94 & 1.16 & 0.75 & 0.53 \\
\hline-80.53 & 0.66 & 1.61 & 2.6 & 3 & 4.58 & 4.96 & 4.64 & 3.24 & 1.99 & 1.18 & 0.77 & 0.54 \\
\hline-78.63 & 0.67 & 1.64 & 2.65 & 2.95 & 4.43 & 4.89 & 4.52 & 3.11 & 2.04 & 1.21 & 0.79 & 0.55 \\
\hline-76.74 & 0.69 & 1.69 & 2.7 & 2.9 & 4.26 & 4.8 & 4.38 & 2.99 & 2.06 & 1.26 & 0.82 & 0.56 \\
\hline-74.84 & 0.71 & 1.76 & 2.73 & 2.85 & 4.05 & 4.69 & 4.2 & 2.89 & 2.08 & 1.3 & 0.85 & 0.58 \\
\hline-72.95 & 0.73 & 1.85 & 2.74 & 2.82 & 3.84 & 4.54 & 4 & 2.82 & 2.08 & 1.36 & 0.9 & 0.61 \\
\hline-71.05 & 0.76 & 1.94 & 2.75 & 2.8 & 3.67 & 4.34 & 3.79 & 2.78 & 2.08 & 1.41 & 0.95 & 0.64 \\
\hline-69.16 & 0.8 & 2.03 & 2.74 & 2.79 & 3.55 & 4.13 & 3.6 & 2.76 & 2.08 & 1.47 & 1.01 & 0.68 \\
\hline-67.26 & 0.85 & 2.12 & 2.72 & 2.78 & 3.47 & 3.95 & 3.47 & 2.72 & 2.08 & 1.52 & 1.09 & 0.72 \\
\hline-65.37 & 0.91 & 2.2 & 2.71 & 2.76 & 3.4 & 3.83 & 3.39 & 2.69 & 2.08 & 1.57 & 1.18 & 0.78 \\
\hline-63.47 & 1 & 2.27 & 2.7 & 2.75 & 3.35 & 3.76 & 3.33 & 2.66 & 2.08 & 1.62 & 1.28 & 0.86 \\
\hline-61.58 & 1.11 & 2.34 & 2.69 & 2.73 & 3.31 & 3.69 & 3.26 & 2.63 & 2.08 & 1.66 & 1.38 & 0.95 \\
\hline-59.68 & 1.23 & 2.41 & 2.67 & 2.71 & 3.26 & 3.63 & 3.2 & 2.6 & 2.08 & 1.7 & 1.47 & 1.06 \\
\hline-57.79 & 1.34 & 2.47 & 2.65 & 2.69 & 3.23 & 3.57 & 3.13 & 2.56 & 2.07 & 1.73 & 1.55 & 1.17 \\
\hline-55.89 & 1.46 & 2.52 & 2.63 & 2.66 & 3.19 & 3.5 & 3.05 & 2.51 & 2.06 & 1.75 & 1.62 & 1.29 \\
\hline-54.00 & 1.57 & 2.55 & 2.6 & 2.64 & 3.15 & 3.43 & 2.97 & 2.47 & 2.04 & 1.75 & 1.68 & 1.41 \\
\hline-52.11 & 1.68 & 2.59 & 2.58 & 2.61 & 3.1 & 3.35 & 2.89 & 2.43 & 2.02 & 1.75 & 1.73 & 1.52 \\
\hline-50.21 & 1.79 & 2.61 & 2.56 & 2.58 & 3.05 & 3.27 & 2.81 & 2.38 & 2 & 1.75 & 1.76 & 1.63 \\
\hline-48.32 & 1.89 & 2.64 & 2.54 & 2.55 & 2.99 & 3.18 & 2.73 & 2.33 & 1.98 & 1.74 & 1.78 & 1.72 \\
\hline-46.42 & 1.98 & 2.67 & 2.51 & 2.52 & 2.93 & 3.1 & 2.65 & 2.27 & 1.95 & 1.72 & 1.79 & 1.8 \\
\hline-44.53 & 2.05 & 2.68 & 2.49 & 2.49 & 2.86 & 3.03 & 2.58 & 2.22 & 1.93 & 1.71 & 1.8 & 1.87 \\
\hline-42.63 & 2.11 & 2.67 & 2.46 & 2.46 & 2.79 & 2.96 & 2.51 & 2.17 & 1.91 & 1.7 & 1.8 & 1.93 \\
\hline-40.74 & 2.16 & 2.65 & 2.44 & 2.43 & 2.72 & 2.88 & 2.45 & 2.13 & 1.89 & 1.69 & 1.8 & 1.98 \\
\hline-38.84 & 2.2 & 2.61 & 2.42 & 2.39 & 2.66 & 2.81 & 2.39 & 2.08 & 1.88 & 1.69 & 1.81 & 2.03 \\
\hline-36.95 & 2.21 & 2.57 & 2.39 & 2.36 & 2.59 & 2.74 & 2.33 & 2.04 & 1.86 & 1.69 & 1.82 & 2.06 \\
\hline-35.05 & 2.22 & 2.53 & 2.37 & 2.33 & 2.53 & 2.67 & 2.28 & 2.01 & 1.86 & 1.69 & 1.83 & 2.08 \\
\hline-33.16 & 2.21 & 2.48 & 2.34 & 2.31 & 2.47 & 2.6 & 2.24 & 1.98 & 1.85 & 1.69 & 1.84 & 2.1 \\
\hline-31.26 & 2.19 & 2.44 & 2.32 & 2.29 & 2.42 & 2.53 & 2.2 & 1.95 & 1.85 & 1.7 & 1.85 & 2.1 \\
\hline-29.37 & 2.17 & 2.39 & 2.3 & 2.26 & 2.37 & 2.46 & 2.16 & 1.93 & 1.85 & 1.71 & 1.86 & 2.11 \\
\hline-27.47 & 2.15 & 2.34 & 2.28 & 2.24 & 2.31 & 2.4 & 2.13 & 1.92 & 1.85 & 1.73 & 1.87 & 2.11 \\
\hline-25.58 & 2.13 & 2.3 & 2.25 & 2.22 & 2.26 & 2.35 & 2.11 & 1.9 & 1.86 & 1.75 & 1.88 & 2.1 \\
\hline-23.68 & 2.1 & 2.25 & 2.23 & 2.2 & 2.22 & 2.3 & 2.09 & 1.9 & 1.88 & 1.78 & 1.89 & 2.09 \\
\hline-21.79 & 2.08 & 2.21 & 2.2 & 2.18 & 2.18 & 2.26 & 2.07 & 1.9 & 1.9 & 1.8 & 1.9 & 2.08 \\
\hline-19.89 & 2.06 & 2.17 & 2.16 & 2.16 & 2.15 & 2.23 & 2.06 & 1.9 & 1.91 & 1.82 & 1.91 & 2.06 \\
\hline-18.00 & 2.03 & 2.13 & 2.13 & 2.14 & 2.11 & 2.2 & 2.06 & 1.91 & 1.93 & 1.84 & 1.92 & 2.05 \\
\hline-16.11 & 2 & 2.09 & 2.09 & 2.11 & 2.08 & 2.17 & 2.05 & 1.91 & 1.95 & 1.86 & 1.92 & 2.04 \\
\hline-14.21 & 1.97 & 2.06 & 2.06 & 2.09 & 2.05 & 2.15 & 2.05 & 1.92 & 1.96 & 1.88 & 1.93 & 2.02 \\
\hline-12.32 & 1.95 & 2.02 & 2.03 & 2.06 & 2.02 & 2.13 & 2.05 & 1.93 & 1.97 & 1.9 & 1.94 & 2.01 \\
\hline-10.42 & 1.92 & 1.99 & 2 & 2.04 & 1.99 & 2.11 & 2.05 & 1.93 & 1.99 & 1.92 & 1.95 & 1.99 \\
\hline
\end{tabular}




\begin{tabular}{|c|c|c|c|c|c|c|c|c|c|c|c|c|}
\hline-8.53 & 1.9 & 1.96 & 1.98 & 2.01 & 1.96 & 2.09 & 2.05 & 1.94 & 2 & 1.94 & 1.95 & 1.98 \\
\hline-6.63 & 1.87 & 1.93 & 1.95 & 1.98 & 1.94 & 2.08 & 2.05 & 1.95 & 2.01 & 1.95 & 1.96 & 1.97 \\
\hline-4.74 & 1.85 & 1.89 & 1.92 & .95 & 1.92 & 2.07 & 2.05 & 1.96 & 2.02 & 1.97 & 1.97 & 1.95 \\
\hline-2.84 & 1.83 & 1.86 & 1.9 & .91 & 1.9 & .06 & 2.05 & 1.97 & 2.04 & 1.99 & 1.97 & .94 \\
\hline-0.95 & 1.81 & 1.84 & 1.88 & .88 & 1.89 & .05 & 2.06 & 1.98 & 2.05 & 2.01 & 1.98 & .93 \\
\hline 0.95 & 1.8 & 1.82 & 1.86 & & .87 & .04 & .06 & 1.99 & 2.06 & 2.02 & 1.98 & .91 \\
\hline 2.84 & 1.79 & 1.8 & 1.84 & .83 & 1.85 & .03 & .06 & 2 & 2.06 & 2.02 & 1.99 & 1.9 \\
\hline 4.74 & 1.78 & 1.78 & 1.82 & 1.8 & 1.83 & .02 & .07 & 2.01 & 2.07 & 2.02 & 1.99 & 1.9 \\
\hline 6.63 & 1.78 & 1.77 & 1.8 & 1.78 & 1.82 & .01 & .08 & 2.01 & 2.07 & 2.02 & & 1.9 \\
\hline 8.53 & 1.78 & 1.77 & 1.78 & .76 & 1.8 & .02 & .09 & 2.02 & 2.07 & 2.02 & 2.01 & 1.91 \\
\hline .42 & 1.79 & 1.76 & 1.76 & 1.74 & 1.79 & 2.02 & .11 & 2.04 & 2.08 & 2.04 & 2.03 & 1.92 \\
\hline 2.32 & 1.81 & 1.76 & 1.74 & 1.72 & 1.78 & 2.03 & 2.14 & 2.07 & 2.1 & 2.07 & 2.06 & 1.94 \\
\hline .21 & 1.82 & 1.76 & 1.72 & 1.7 & 1.76 & 2.03 & 2.16 & 2.11 & 2.12 & 2.1 & 2.08 & 1.96 \\
\hline .11 & 1.84 & 1.76 & 1.7 & 1.68 & 1.74 & 2.03 & 2.19 & 2.15 & 2.15 & 2.12 & 2.11 & 1.98 \\
\hline .00 & 1.86 & 1.76 & 1.68 & 66 & 1.73 & & 2.22 & 2.19 & 2.17 & 2.15 & 2.14 & .01 \\
\hline .89 & 1.88 & 1.75 & 1.66 & .63 & 1.71 & 2.02 & 2.24 & 2.22 & 2.19 & 2.17 & 2.16 & 2.03 \\
\hline .79 & 1.9 & 1.75 & 1.64 & & 1.69 & 2.01 & 2.26 & 2.25 & 2.2 & 2.19 & 2.19 & 2.06 \\
\hline .68 & 1.93 & 1.75 & 1.62 & .59 & 1.67 & 2 & 2.28 & 2.28 & 2.21 & 2.2 & 2.22 & 2.09 \\
\hline .58 & 1.95 & 1.75 & 1.59 & 56 & 1.65 & 1.98 & 2.3 & 2.31 & 2.22 & 2.21 & 2.24 & 2.12 \\
\hline 7.47 & 1.98 & 1.75 & 1.57 & 1.54 & 1.63 & 1.96 & 2.31 & 2.34 & 2.22 & 2.22 & 2.26 & 2.16 \\
\hline .37 & 2.02 & 1.75 & 1.56 & .52 & 1.61 & 1.94 & 2.32 & 2.37 & 2.23 & 2.22 & 2.28 & 2.21 \\
\hline 1.26 & 2.05 & 1.75 & 1.54 & .49 & 1.59 & 1.92 & 2.31 & 2.4 & 2.24 & 2.22 & 2.3 & 2.25 \\
\hline 3.16 & 2.1 & 1.76 & 1.52 & .47 & 1.58 & 1.89 & 2.3 & 2.43 & 2.23 & 2.22 & 2.31 & 2.3 \\
\hline .05 & 2.15 & 1.77 & 1.51 & .45 & 1.56 & 1.85 & 2.27 & 2.46 & 2.23 & 2.21 & 2.33 & 2.35 \\
\hline .95 & 2.19 & 1.78 & 1.49 & 1.43 & 1.55 & 1.81 & 2.23 & 2.48 & 2.22 & 2.21 & 2.34 & 2.4 \\
\hline 8.84 & 2.25 & 1.8 & 1.48 & 1.41 & 1.54 & 1.77 & 2.17 & 2.5 & 2.22 & 2.21 & 2.37 & 2.46 \\
\hline 0.74 & 2.31 & 1.82 & 1.47 & 1.4 & 1.53 & 1.72 & 2.09 & 2.52 & 2.22 & 2.21 & 2.39 & 2.51 \\
\hline 2.63 & 2.36 & 1.84 & 1.46 & 1.38 & 1.52 & 1.66 & 2.01 & 2.52 & 2.22 & 2.22 & 2.41 & 2.57 \\
\hline 4.53 & 2.42 & 1.87 & 1.46 & .36 & 1.51 & 1.59 & 1.92 & 2.51 & 2.23 & 2.22 & 2.43 & 2.63 \\
\hline & 2.46 & 1.89 & 1.46 & & 1.5 & 1.52 & 1.81 & 2.49 & 2.23 & 2.23 & 2.45 & 2.69 \\
\hline 837 & 2.5 & 1.92 & 1.46 & .33 & 1.49 & 1.45 & 1.71 & 2.47 & 2.25 & 2.24 & 2.48 & 2.75 \\
\hline 0.21 & 2.54 & 1.94 & 1.46 & 1.32 & 1.47 & 1.38 & 1.62 & 2.43 & 2.26 & 2.24 & 2.5 & 2.8 \\
\hline 11 & 2.57 & 1.96 & 1.46 & 1.3 & 1.45 & 1.31 & 1.53 & 2.38 & 2.27 & 2.24 & 2.53 & 2.85 \\
\hline 4.00 & 2.6 & 1.98 & 1.46 & 1.28 & 1.44 & 1.25 & 1.45 & 2.34 & 2.29 & 2.24 & 2.55 & 2.9 \\
\hline 5.89 & 2.63 & 1.99 & 1.47 & 1.26 & 1.41 & 1.19 & 1.37 & 2.28 & 2.3 & 2.24 & 2.57 & 2.94 \\
\hline 7.79 & 2.64 & 2 & 1.47 & 1.25 & 1.39 & 1.13 & 1.29 & 2.22 & 2.3 & 2.23 & 2.58 & 2.97 \\
\hline 9.68 & 2.66 & 2.01 & 1.48 & 1.22 & 1.37 & 1.07 & 1.21 & 2.16 & 2.31 & 2.23 & 2.6 & 2.99 \\
\hline 1.58 & 2.67 & 2.02 & 1.48 & 1.2 & 1.34 & 1.01 & 1.13 & 2.1 & 2.31 & 2.23 & 2.62 & 3.02 \\
\hline 3.47 & 2.7 & 2.03 & 1.48 & 1.18 & 1.31 & 0.96 & 1.07 & 2.03 & 2.31 & 2.22 & 2.64 & 3.0 \\
\hline 5.37 & 2.72 & 2.04 & 1.48 & 1.15 & 1.27 & 0.93 & 1.01 & 1.95 & 2.31 & 2.21 & 2.66 & 3.07 \\
\hline 67.26 & 2.75 & 2.05 & 1.48 & 1.13 & 1.24 & 0.9 & 0.97 & 1.88 & 2.31 & 2.2 & 2.69 & 3.11 \\
\hline 69.16 & 2.82 & 2.07 & 1.47 & 1.1 & 1.22 & 0.88 & 0.95 & 1.8 & 2.3 & 2.2 & 2.72 & 3.19 \\
\hline & 2.91 & 2.07 & 1.46 & 1.07 & 1.2 & 0.86 & 0.92 & 1.74 & 2.3 & 2.2 & 2.77 & 3.28 \\
\hline 72.95 & 3.03 & 2.08 & 1.45 & 1.05 & 1.19 & 0.84 & 0.9 & 1.69 & 2.29 & 2.2 & 2.83 & 3.3 \\
\hline & 3.15 & 2.09 & 1.43 & 1.02 & 1.18 & 0.82 & 0.89 & 1.66 & 2.28 & 2.21 & 2.92 & 3.42 \\
\hline 76.74 & 3.26 & 2.1 & 1.41 & 1.01 & 1.17 & 0.81 & 0.88 & 1.63 & 2.25 & 2.23 & 3.03 & 3.47 \\
\hline
\end{tabular}




\begin{tabular}{rrrrrrrrrrrrr}
78.63 & 3.35 & 2.14 & 1.4 & 1 & 1.15 & 0.79 & 0.87 & 1.62 & 2.22 & 2.25 & 3.14 & 3.51 \\
80.53 & 3.43 & 2.19 & 1.38 & 0.99 & 1.14 & 0.78 & 0.86 & 1.62 & 2.19 & 2.29 & 3.25 & 3.55 \\
82.42 & 3.5 & 2.27 & 1.35 & 0.99 & 1.13 & 0.78 & 0.85 & 1.61 & 2.16 & 2.33 & 3.35 & 3.58 \\
84.32 & 3.57 & 2.34 & 1.33 & 0.99 & 1.12 & 0.77 & 0.84 & 1.6 & 2.14 & 2.39 & 3.43 & 3.62 \\
86.21 & 3.62 & 2.39 & 1.31 & 0.99 & 1.11 & 0.77 & 0.83 & 1.59 & 2.12 & 2.44 & 3.49 & 3.66 \\
88.11 & 3.66 & 2.43 & 1.29 & 0.99 & 1.11 & 0.76 & 0.83 & 1.58 & 2.1 & 2.48 & 3.52 & 3.7 \\
90.00 & 3.69 & 2.44 & 1.29 & 0.99 & 1.11 & 0.76 & 0.83 & 1.57 & 2.1 & 2.51 & 3.54 & 3.72 \\
\hline
\end{tabular}


Table S5. Monthly mean $\mathrm{Ca}^{+}$column abundance $(70-110 \mathrm{~km})$ predicted by WACCM-Ca (units: $10^{7}$ atom $\mathrm{cm}^{-2}$ )

\begin{tabular}{|c|c|c|c|c|c|c|c|c|c|c|c|c|}
\hline Latitude & Jan & Feb & Mar & Apr & May & Jun & Jul & Aug & Sep & Oct & Nov & Dec \\
\hline-90.00 & 15.3 & 37.2 & 35.3 & 26.8 & 32.3 & 33.7 & 33.4 & 27.5 & 22.5 & 17.6 & 16.3 & 11.9 \\
\hline & 15.3 & 37.2 & 35.2 & 26.8 & 32.2 & 33.6 & 33.3 & 27.4 & & & & \\
\hline 5.21 & 15.4 & 37.3 & 34.9 & 26.7 & 32.0 & 33.5 & 33.2 & 27.2 & 22.3 & 17.6 & & 12.0 \\
\hline & 15.5 & 37.5 & 34.6 & 26.6 & 31.6 & 33.2 & 33.0 & 27.0 & 22.2 & 17.6 & 16.6 & 12.1 \\
\hline & 15.7 & 37.7 & 34.2 & 26.4 & 31.2 & 33.0 & 32.6 & 26.7 & 22.0 & 17.7 & & 12.4 \\
\hline .53 & 16.1 & 38.0 & 33.7 & 26.2 & 30.8 & 32.7 & 32.2 & 26.5 & 21.8 & 17.9 & 17.3 & 12.7 \\
\hline & 16.5 & 38.3 & 33.1 & 26.0 & 30.5 & 32.5 & 31.8 & 26.2 & 21.7 & 18.0 & 17.7 & 13.1 \\
\hline & 17.0 & 38.6 & 32.4 & 25.7 & 30.2 & 32.2 & 31.4 & 26.0 & 21.6 & 18.2 & 18.3 & 13.6 \\
\hline & 17.6 & 39.0 & 31.6 & 25.4 & 29.9 & 32.0 & 31.0 & 25.7 & 21.5 & & 18.9 & 14.2 \\
\hline & & 39.2 & 30.9 & 25.0 & 29.5 & 31.9 & 30.7 & 25.4 & 21.4 & & 19.6 & 14.9 \\
\hline .05 & 19.0 & 39.5 & 30.2 & 24.8 & 29.1 & 31.8 & 30.3 & 25.1 & 21.3 & 18.8 & 20.4 & 15.8 \\
\hline & 9.9 & 39.7 & 29.5 & 24.5 & 28.7 & 31.8 & 29.9 & 24.8 & 21.3 & & 21.3 & 16.8 \\
\hline .26 & 21.0 & 39.9 & 28.8 & 24.3 & 28.3 & 31.6 & 29.4 & 24.5 & 21.2 & 19.3 & 22.2 & 18.0 \\
\hline & 2.3 & 40.1 & 28.3 & 24.2 & 28.0 & 31.3 & 28.9 & 24.3 & 21.2 & & 23.1 & 19.4 \\
\hline .47 & 23.8 & 40.3 & 27.9 & 24.0 & 27.7 & 31.0 & 28.4 & 24.1 & 21.2 & 19.7 & 24.0 & 20.9 \\
\hline & .3 & 40.4 & 27.6 & 23.7 & 27.4 & 30.6 & 28.1 & & 21.1 & & 24.7 & 22.6 \\
\hline & 26.9 & 40.6 & 27.2 & 23.6 & 27.2 & 30.3 & 27.7 & 23.7 & 21.0 & 19.9 & 25.3 & 24.3 \\
\hline & 28.5 & 40.7 & 26.8 & 23.4 & 27.0 & 30.1 & 27.4 & & 20.9 & & & 26.0 \\
\hline & 30.0 & 40.7 & 26.5 & 23.2 & 26.9 & 29.9 & 27.0 & 23.2 & 20.8 & .8 & 26.0 & 27.6 \\
\hline .00 & 31.4 & 40.7 & 26.2 & 23.1 & 26.7 & 29.7 & 26.6 & 22.9 & 20.7 & 19.7 & 26.0 & 29.0 \\
\hline & 2.7 & 40.5 & 25.9 & 23.0 & 26.6 & 29.5 & 26.2 & 22.6 & 20.5 & .5 & 25.9 & 30.2 \\
\hline 21 & 33 & 40.1 & 25.8 & 22.9 & 26.5 & 29.3 & 25.8 & 22.3 & 20.4 & 19.3 & 25.7 & 31.0 \\
\hline & 34.5 & 39.6 & 25.6 & 22.8 & 26.3 & 29.0 & 25.3 & 22.0 & 20.3 & 19.1 & 25.3 & 31.6 \\
\hline & 34.9 & 39.1 & 25.4 & 22.8 & 26.1 & 28.8 & 24.9 & 21.7 & 20.1 & 19.0 & 25.0 & 32.0 \\
\hline & & & & & & & & & 20.0 & & & 32.2 \\
\hline & 35.1 & 37.5 & 25.0 & 22.6 & 25.5 & 28.3 & 24.0 & 21.0 & 19.9 & 18.7 & 24.4 & 32.2 \\
\hline & & 36.4 & 24.9 & 22.6 & 25.2 & 28.0 & 23.6 & & 19.8 & & 24.1 & 32.2 \\
\hline & 34.3 & 35.2 & 24.8 & 22.5 & 24.9 & 27.7 & 23.2 & 20.4 & 19.7 & & 23.9 & 32.0 \\
\hline & & & 24.7 & 22.5 & 24.7 & 27.4 & 22.8 & 20.1 & 19.6 & & 23.8 & 31.7 \\
\hline .05 & 3.0 & 32.9 & 24.6 & 22.5 & 24.4 & 27.0 & 22.4 & 19.9 & 19.6 & 18.6 & 23.7 & 31.4 \\
\hline & & 31.7 & 24.6 & 22.4 & 24.2 & 26.5 & 22.2 & 19.8 & 19.6 & & 23.6 & 30.9 \\
\hline-31.26 & 31.5 & 30.7 & 24.5 & 22.4 & 24.0 & 26.1 & 21.9 & 19.7 & 19.7 & 18.8 & 23.6 & 30.3 \\
\hline & .6 & 29.7 & 24.5 & 22.4 & 23.7 & 25.7 & 21.7 & 19.6 & 19.8 & 19.0 & 23.6 & 29.7 \\
\hline & & 28.7 & 24.4 & 22.5 & 23.5 & 25.4 & 21.5 & & 20.0 & 19.2 & 23.6 & 29.1 \\
\hline & 29.0 & 27.8 & 24.4 & 22.5 & 23.3 & 25.0 & 21.4 & 19.6 & 20.2 & 19.6 & 23.6 & 28.4 \\
\hline & & 27.0 & 24.3 & 22.6 & 23.1 & 24.7 & 21.4 & 19.7 & 20.5 & 19.9 & 23.5 & 27.7 \\
\hline & 27.5 & 26.3 & 24.1 & 22.7 & 23.0 & 24.5 & 21.4 & 19.9 & 20.8 & 20.3 & 23.5 & 27.1 \\
\hline & 26.8 & 25.7 & 24.0 & 22.8 & 22.9 & 24.4 & 21.5 & 20.1 & 21.2 & 20.7 & 23.5 & 26.4 \\
\hline-18.00 & 26.1 & 25.1 & 23.8 & 22.9 & 22.8 & 24.2 & 21.7 & 20.3 & 21.6 & 21.1 & 23.5 & 25.8 \\
\hline & 25.4 & 24.6 & 23.6 & 22.9 & 22.7 & 24.1 & 21.8 & 20.5 & 21.9 & 21.4 & 23.4 & 25.3 \\
\hline-14.21 & 24.8 & 24.1 & 23.4 & 22.9 & 22.6 & 24.1 & 22.0 & 20.8 & 22.2 & 21.7 & 23.3 & 24.8 \\
\hline & 24.2 & 23.6 & 23.2 & 22.9 & 22.6 & 24.0 & 22.2 & 21.0 & 22.5 & 22.0 & 23.3 & 24.3 \\
\hline-10.42 & 23.6 & 23.1 & 23.1 & 22.8 & 22.5 & 24.0 & 22.4 & 21.3 & 22.9 & 22.3 & 23.2 & 23.8 \\
\hline
\end{tabular}




\begin{tabular}{|c|c|c|c|c|c|c|c|c|c|c|c|c|}
\hline-8.53 & 23.1 & 22.7 & 22.9 & 22.8 & 22.4 & 23.9 & 22.6 & 21.5 & 23.2 & 22.6 & 23.2 & 23.4 \\
\hline-6.63 & 22.6 & 22.2 & 22.8 & 22.6 & 22.4 & 23.9 & 22.7 & 21.7 & 23.4 & 22.9 & 23.2 & 23.0 \\
\hline-4.74 & 22.1 & 21.8 & 22.6 & 22.4 & 22.3 & 24.0 & 22.9 & 21.9 & 23.6 & 23.1 & 23.1 & 22.7 \\
\hline-2.84 & 21.7 & 21.3 & 22.4 & 22.2 & 22.3 & 24.0 & 23.0 & 22.1 & 23.9 & 23.4 & 23.0 & 22.3 \\
\hline-0.95 & 21.3 & 20.9 & 22.2 & 21.9 & 22.3 & 24.0 & 23.2 & 22.2 & 24.0 & 23.5 & 22.9 & 22.0 \\
\hline 0.95 & 20.9 & 20.5 & 21.9 & 21.6 & 22.2 & 24.1 & 23.3 & 22.4 & 24.0 & 23.6 & 22.8 & 21.6 \\
\hline 2.84 & 20.6 & 20.2 & 21.6 & 21.2 & 22.2 & 24.2 & 23.5 & 22.5 & 23.9 & 23.5 & 22.6 & 21.3 \\
\hline 4.74 & 20.4 & 19.9 & 21.2 & 20.9 & 22.1 & 24.2 & 23.7 & 22.5 & 23.8 & 23.3 & 22.4 & 1.1 \\
\hline 6.63 & 0.2 & 19.6 & 20.9 & 20.5 & 22.1 & 24.4 & 23.9 & 22.5 & 23.6 & 3.0 & 22.3 & 20.9 \\
\hline 8.53 & 0.1 & 19.3 & 20.5 & 20.2 & 22.0 & 24.6 & 24.2 & 22.6 & 23.3 & 2.8 & 22.1 & 20.8 \\
\hline 10.42 & 20.0 & 19.1 & 20.1 & 19.8 & 22.0 & 24.9 & 24.5 & 22.8 & 23.2 & 22.7 & 22.1 & 20.8 \\
\hline 12.32 & 19.9 & 18.8 & 19.6 & 19.4 & 21.9 & 25.3 & 25.0 & 23.2 & 23.2 & 22.7 & 22.1 & 20.8 \\
\hline 14.21 & 19.8 & 18.6 & 19.1 & 19.0 & 21.7 & 25.6 & 25.6 & 23.7 & 23.2 & 22.7 & 22.2 & 20.8 \\
\hline 16.11 & 19.8 & 18.3 & 18.6 & 18.5 & 21.5 & 25.8 & 26.1 & 24.1 & 23.2 & 22.7 & 22.2 & 20.8 \\
\hline 18.00 & 19.7 & 18.0 & 18.1 & 18.1 & 21.3 & 26.0 & 26.7 & 24.6 & 23.2 & 22.7 & 22.1 & 20.8 \\
\hline 19.89 & 19.7 & 17.7 & 17.6 & 17.6 & 21.0 & 26.2 & 27.3 & 25.1 & 23.2 & 22.6 & 22.1 & 20.8 \\
\hline 21.79 & 19.6 & 17.4 & 17.0 & 17.1 & 20.7 & 26.3 & 28.0 & 25.6 & 23.2 & 22.4 & 22.0 & 20.8 \\
\hline 23.68 & 19.6 & 17.1 & 16.5 & 16.7 & 20.4 & 26.4 & 28.6 & 26.2 & 23.0 & 22.1 & 21.9 & 20.9 \\
\hline 5.58 & 19.6 & 16.8 & 16.1 & 16.3 & 20.0 & 26.5 & 29.3 & 26.7 & 22.9 & 21.8 & 21.8 & 20.9 \\
\hline 27.47 & 19.6 & 16.5 & 15.6 & 15.8 & 19.7 & 26.5 & 29.9 & 27.3 & 22.7 & 21.5 & 21.6 & 21.0 \\
\hline 29.37 & 19.6 & 16.2 & 15.2 & 15.4 & 19.4 & 26.6 & & 28.0 & & 21.1 & 21.5 & 21.2 \\
\hline 31.26 & 19.7 & 16.0 & 14.8 & 15.1 & 19.1 & 26.5 & 31.2 & 28.8 & 22.3 & 20.7 & 21.3 & 21.3 \\
\hline 33.16 & 19.8 & 15.8 & 14.4 & 14.7 & 18.8 & 26.4 & 31.6 & 29.6 & 22.1 & 20.3 & 21.1 & 21.5 \\
\hline 35.05 & 19.9 & 15.7 & 14.1 & 14.4 & 18.6 & 26.2 & 31.9 & 30.4 & 21.8 & 20.0 & 20.9 & 21.7 \\
\hline 36.95 & 20.1 & 15.6 & 13.8 & 14.1 & 18.4 & 25.9 & 32.0 & 31.1 & 21.5 & 19.6 & 20.7 & 21.9 \\
\hline 38.84 & 20.3 & 15.6 & 13.5 & 13.8 & 18.2 & 25.5 & 31.8 & 31.8 & 21.3 & 19.3 & 20.5 & 22.1 \\
\hline 40.74 & 20.5 & 15.7 & 13.3 & 13.6 & 18.1 & 25.0 & 31.4 & 32.4 & 21.1 & 19.0 & 20.4 & 22.3 \\
\hline 42.63 & 20.8 & 15.7 & 13.1 & 13.3 & 18.0 & 24.4 & 30.6 & 33.0 & 21.0 & 18.9 & 20.2 & 22.5 \\
\hline 44.53 & 20.9 & 15.8 & 13.0 & 13.1 & 18.0 & 23.8 & 29.6 & 33.3 & 20.9 & 18.7 & 20.1 & 22.7 \\
\hline 46.42 & 21.0 & & & & 18.0 & 23.1 & 28.5 & 33.6 & 20.9 & 18.5 & 19.9 & 22.8 \\
\hline 48.32 & 21.1 & 16.0 & 12.8 & 12.8 & 18.0 & 22.4 & 27.3 & 33.8 & 21.1 & 18.3 & 19.8 & 23.0 \\
\hline 50.21 & 21.1 & 16.1 & 12.7 & 12.7 & 18.0 & 21.7 & 26.1 & 33.9 & 21.2 & 18.2 & 19.8 & 23.1 \\
\hline 52.11 & 21.1 & 16.2 & 12.7 & 12.5 & 18.0 & 21.0 & 25.0 & 34.0 & 21.4 & 18.0 & 19.7 & 23.2 \\
\hline 54.00 & 21.1 & 16.2 & 12.7 & 12.4 & 18.0 & 20.4 & 23.9 & 34.0 & 21.7 & 17.9 & 19.7 & 23.2 \\
\hline 55.89 & 21.0 & 16.2 & 12.7 & 12.2 & 18.0 & 19.8 & 22.9 & 33.9 & 21.9 & 17.8 & 19.6 & 23.2 \\
\hline 57.79 & 21.0 & 16.3 & 12.8 & 12.1 & 18.1 & 19.3 & 22.0 & 33.8 & 22.1 & 17.6 & 19.6 & 23.2 \\
\hline 59.68 & 20.9 & 16.4 & 12.9 & 12.0 & 18.2 & 18.9 & 21.2 & 33.7 & 22.3 & 17.5 & 19.5 & 23.2 \\
\hline 61.58 & 20.9 & 16.5 & 12.9 & 11.9 & 18.3 & 18.5 & 20.4 & 33.4 & 22.6 & 17.5 & 19.5 & 23.2 \\
\hline 63.47 & 21.1 & 16.6 & 13.0 & 11.8 & 18.5 & 18.1 & 19.8 & 33.1 & 22.8 & 17.5 & 19.5 & 23.3 \\
\hline 65.37 & 21.2 & 16.7 & 13.0 & 11.8 & 18.7 & 17.8 & 19.3 & 32.9 & 23.1 & 17.4 & 19.6 & 23.3 \\
\hline 67.26 & 21.4 & 16.9 & 13.1 & 11.8 & 18.9 & 17.5 & 18.8 & 32.6 & 23.4 & 17.4 & 19.7 & 23.4 \\
\hline 69.16 & 21.7 & 17.0 & 13.2 & 11.9 & 19.1 & 17.3 & 18.4 & 32.4 & 23.8 & 17.5 & 19.8 & 23.2 \\
\hline 71.05 & 21.8 & 17.2 & 13.3 & 12.0 & 19.2 & 17.1 & 18.0 & 32.2 & 24.3 & 17.6 & 20.0 & 23.1 \\
\hline 72.95 & 22.0 & 17.4 & 13.4 & 12.1 & 19.3 & 16.9 & 17.7 & 32.2 & 24.9 & 17.9 & 20.1 & 23.0 \\
\hline 74.84 & 22.2 & 17.6 & 13.5 & 12.3 & 19.3 & 16.7 & 17.5 & 32.2 & 25.5 & 18.1 & 20.2 & 22.9 \\
\hline 76.74 & 22.5 & 17.7 & 13.6 & 12.4 & 19.3 & 16.5 & 17.3 & 32.2 & 26.1 & 18.5 & 20.3 & 22.8 \\
\hline
\end{tabular}




\begin{tabular}{lllllllllllll}
78.63 & 22.7 & 17.8 & 13.8 & 12.5 & 19.3 & 16.3 & 17.1 & 32.2 & 26.6 & 18.8 & 20.4 & 22.8 \\
80.53 & 23.1 & 17.8 & 14.0 & 12.6 & 19.3 & 16.2 & 17.0 & 32.2 & 27.1 & 19.1 & 20.5 & 22.8 \\
82.42 & 23.4 & 17.8 & 14.3 & 12.6 & 19.3 & 16.1 & 16.9 & 32.2 & 27.5 & 19.4 & 20.7 & 22.9 \\
84.32 & 23.6 & 17.9 & 14.5 & 12.7 & 19.3 & 16.1 & 16.8 & 32.1 & 27.9 & 19.6 & 20.9 & 23.1 \\
86.21 & 23.8 & 18.0 & 14.6 & 12.7 & 19.3 & 16.1 & 16.7 & 32.0 & 28.1 & 19.7 & 21.1 & 23.3 \\
88.11 & 24.0 & 18.1 & 14.8 & 12.8 & 19.4 & 16.1 & 16.7 & 31.9 & 28.1 & 19.8 & 21.2 & 23.4 \\
90.00 & 24.1 & 18.1 & 14.9 & 12.8 & 19.4 & 16.1 & 16.6 & 31.8 & 28.2 & 19.9 & 21.3 & 23.5 \\
\hline
\end{tabular}




\section{References}

Dawkins, E. C. M., J. M. C. Plane, M. P. Chipperfield, and W. Feng (2015), The nearglobal mesospheric potassium layer: Observations and modeling, J. Geophys. Res. Atmos., 120, 7975-7987.

Frisch, M. J., G. W. Trucks, H. B. Schlegel, G. E. Scuseria, M. A. Robb, J. R. Cheeseman, G. Scalmani, V. Barone, B. Mennucci, G. A. Petersson, et al. (2009), Gaussian 09, edited, Gaussian, Inc., Wallingford, CT, USA.

Gómez-Martín, J. C., and J. M. C. Plane (2017), Reaction Kinetics of $\mathrm{CaOH}$ with $\mathrm{H}$ and $\mathrm{O}_{2}$ and $\mathrm{O}_{2} \mathrm{CaOH}$ with $\mathrm{O}$ : Implications for the Atmospheric Chemistry of Meteoric Calcium, ACS Earth Space Chem., 1, 431-441. 